Sarga MOUSSA

\title{
Un voyage, deux regards : la construction de l'ailleurs oriental chez Lamartine et Delaroière
}

Revivifiée par Chateaubriand avec l'Itinéraire de Paris à Jérusalem (1811), la tradition du pèlerinage en Orient connaît un grand succès à l'époque romantique $^{1}$. Comportant une dimension tout à la fois religieuse et culturelle, ce parcours circulaire de la Méditerranée se veut retour aux origines de la civilisation occidentale, - la Grèce, la Palestine, l'Égypte même. Mais tout en croyant se retrouver en terrain connu, le pèlerin moderne ne peut échapper à l'expérience de la différence. Car l'Orient, au début du XIX ${ }^{\mathrm{e}}$ siècle, c'est aussi l'Empire ottoman (donc un autre système politique), avec sa très grande variété ethnique et confessionnelle, et notamment une présence importante de l'islam qui ne laisse aucun voyageur indifférent. Cette confrontation avec l'altérité orientale, impulsée et souvent médiatisée par Chateaubriand, ne produit pourtant pas les mêmes effets. En ce sens, il est intéressant de comparer deux récits d'un même voyage, celui accompli par Lamartine et par le médecin Delaroière, en 1832-1833. Rappelons-en brièvement les circonstances.

Après un début de carrière diplomatique en Italie, Lamartine démissionne en 1830 de son poste de secrétaire d'ambassade, par fidélité aux Bourbons. C'est la voie ouverte à une carrière politique, que le voyage en Orient servira à asseoir ${ }^{2}$, - Lamartine apprend sa nomination comme député alors qu'il se trouve encore en Syrie, et on trouve parmi ses premières interventions à la Chambre des discours sur la «question d'Orient ${ }^{3}$ ». Lorsqu'il s'embarque à Marseille, en juillet 1832, Lamartine est donc à la fois un poète célèbre (il a publié les Méditations en 1820, les Harmonies en 1830) et un homme politique en devenir, déjà en campagne, comme l'attestent les visites, les banquets, les rituels officiels qui précèdent son départ. Il est par ailleurs accompagné de sa femme Marianne, de sa fille unique Julia (qui mourra de tuberculose à Beyrouth), de plusieurs domestiques, enfin de trois amis, dont l'un, Jean-Vaast Delaroière, médecin et ancien maire d'Hondschoote ${ }^{4}$, avait contribué à la victoire parlementaire de Lamartine. Toute cette petite société loue un brick (c'est l'une des dernières traversées méditerranéennes sur un bateau à voile) qui servira pendant le trajet

\footnotetext{
${ }^{1}$ Voir Jean-Claude Berchet, Le Voyage en Orient, Paris, Laffont, « Bouquins », 1985.

${ }^{2}$ Voir William Fortescue, Alphonse de Lamartine. A Political Biography, Londres, St Martin's Press, 1983, ainsi que Nicolas Courtinat, Philosophie, histoire et imaginaire dans le Voyage en Orient de Lamartine, Paris, Champion, 2003, p. 247 et suiv.

${ }^{3}$ Voir mon article «Lamartine et la "question d'Orient" ", dans L'Orientalisme des saint-simoniens, actes du colloque de l'IMA édités par Michel Levallois et Sarga Moussa, Paris, Maisonneuve et Larose, 2006, p. 75-90.

${ }^{4}$ Sur les liens de Delaroière (mort en 1865) avec Lamartine, voir Henry Cochin, Lamartine et la Flandres, Paris, Plon, 1912, p. 79 et suiv. La comparaison du Voyage en Orient de Lamartine avec celui de Delaroière n'a guère suscité d'intérêt jusqu'ici. On peut cependant renvoyer à l'article de Raymond Warnier, "À propos du Voyage en Orient », in Lamartine. Le Livre du Centenaire, éd. Paul Viallaneix, Paris, Flammarion, 1971.
} 
sur mer. Voyons rapidement l'itinéraire de ce voyage, avec ses principales étapes.

Après deux escales (en Sardaigne et à Malte), l'Alceste aborde au sud du Péloponnèse le 6 août 1832. Bien que la guerre de libération de la Grèce soit terminée, les Turcs sont toujours présents dans l'Attique à cette époque. Athènes n'est pas encore redevenue la capitale d'un État que la plupart des voyageurs ne peuvent s'empêcher de comparer avec l'image de la Grèce antique véhiculée par la culture des humanités. La déception est donc au rendez-vous, comme souvent à partir de cette époque ${ }^{5}$. Un mois plus tard, c'est le débarquement à Beyrouth, où Lamartine et ses proches resteront plusieurs mois, avant et après le pèlerinage à Jérusalem. Là aussi, les circonstances politiques sont particulières, puisqu'Ibrahim, fils de Méhémet-Ali et général en chef de l'armée égyptienne, vient de s'emparer de la Syrie. L'Égypte, au début des années 1830, est à l'apogée de sa puissance militaire et menace directement le sultan, au point que Lamartine croit, à ce moment-là, à un écroulement imminent de l'Empire ottoman. Depuis Beyrouth, les voyageurs se rendent par voie de terre en Palestine (octobre 1832). L'un des temps forts de ce voyage dans le voyage, dont on sent qu'il est investi d'une dimension quasi-initiatique (une épidémie de peste sévit à Jérusalem et rend les déplacements très difficiles), est évidemment la visite du Saint-Sépulcre. Delaroière en ressort bouleversé, conforté de bout en bout dans sa foi, tandis que Lamartine, bien que très ému, se sent plus éloigné que jamais de l'orthodoxie catholique, - le Voyage en Orient sera d'ailleurs mis à l'index par Rome en $1836^{6}$. Alors qu'il avait prévu d'aller en Égypte depuis la Terre sainte, Lamartine y renonce, ayant reçu des nouvelles alarmantes concernant la santé de sa fille, restée à Beyrouth. Après la mort de Julia, il part avec sa femme et ses amis, en mars 1833, pour Baalbek et Damas, puis, ayant traversé la chaîne de l'Anti-Liban, il s'embarque en avril pour Constantinople, où il arrive quelques semaines plus tard, après avoir fait escale à Rhodes et à Smyrne. Un mois et demi de séjour dans la capitale ottomane, où les voyaeurs reprennent des forces et admirent les paysages enchanteurs de la Corne d'Or, constitue la dernière grande étape de ce long périple, avant le retour par la Turquie d'Europe (Bulgarie et Serbie, déjà agitées par des mouvements indépendantistes).

Dès son retour en France, en automne 1833, Lamartine pense à publier un ouvrage tiré de ses notes (beaucoup plus travaillées qu'on ne l'imaginerait ${ }^{7}$ ) : ce sera Impressions, souvenirs, pensées et paysages pendant un voyage en Orient..., qui parait en 4 volumes en 1835 et qui devient rapidement, au fil des nombreuses rééditions, Voyage en Orient. Quant à Delaroière, il reprend le

\footnotetext{
${ }^{5}$ Voir Sophie Basch, Le Mirage grec. La Grèce contemporaine devant l'opinion française (1846-1946), Hatier, 1995.

${ }^{6}$ Sur l'évolution de la pensée religieuse de Lamartine à l'époque du Voyage en Orient, voir Henri Guillemin, Le Jocelyn de Lamartine, Paris, Boivin, 1936, p. 209-217.

${ }^{7}$ Voir à ce sujet les carnets de voyage édités par Lotfy Fam, Lamartine, Voyage en Orient, Paris, Nizet, s.d. [1959].
} 
même titre, pour publier en 1836 son propre récit de voyage. Examinons, pour commencer, la façon dont ces deux auteurs décrivent quelques-unes des principales étapes de leur voyage en Orient.

\section{La construction des différences}

Le Parthénon, surtout depuis que Chateaubriand en avait célébré la beauté, fait partie des temps forts de la visite d'Athènes. Mais pour Delaroière comme pour Lamartine, la première vue de ce temple est décevante. L'un et l'autre insistent sur une image préexistante, jugée idéalisante, alors qu'ils voient un ensemble de ruines désarticulées et sans grandeur : « Une petite colline qu'on nomme l'acropole, enfermée dans un mur, couverte de débris de marbre, de quelques colonnes isolées encore debout et de quelques autres, soutenant un reste de temple ; le tout n'offrant pas une idée plus grandiose que la colline même sur laquelle ces restes se trouvent ", écrit Delaroière ${ }^{8}$. Le sentiment de désillusion face à la Grèce moderne portant les stigmates de la guerre est largement partagé par les voyageurs des années 1830. Mais, pour ce qui concerne spécifiquement le Parthénon, on peut supposer que Delaroière s'inspire du Voyage en Orient de Lamartine, qui écrivait de son côté :

L'effet de cet édifice, le plus beau que la main humaine ait élevé sur la terre, au jugement de tous les âges, ne répond en rien à ce qu'on attend, vu ainsi ; et les pompeuses paroles des voyageurs, peintres ou poètes, vous retombent tristement sur le cœur quand vous voyez cette réalité si loin de leurs images 9 .

Malgré cette probable intertextualité, on note aussi des différences de perception dont le récit de chacun des voyageurs porte la trace. Ou, plus exactement, après ce premier moment marqué par la déception (la Grèce moderne, sans pour autant être exotique, apparaît dans une altérité dérangeante), l'un et l'autre se réfugient dans le passé, mais ils trahissent une vision opposée de la démocratie athénienne. Pour Delaroière, le Pnyx (l'Assemblée athénienne) est vue à travers le filtre platonicien de la critique des sophistes, ce qui donne lieu, en retour, à une pique contre les dangers de la démagogie dans la France de Louis-Philippe :

Et ce peuple aveugle dans son amour comme dans sa haine décerna la louange ou le blâme par caprice ou par vanité. Il y a bien des années écoulées depuis lors ; maintenant le peuple est-il plus sage ? plus juste? Les motifs de ceux qui le gouvernent ou qui voudraient le gouverner sont-ils plus purs ? plus désintéressés? Notre future histoire nous l'apprendra ${ }^{10}$.

Doit-on lire, ici, une critique implicite du député-poète, à travers ce discrédit jeté sur la fonction parlementaire? C'est peu probable, même si cette réflexion de

\footnotetext{
${ }^{8}$ Jean-Vaast Delaroière, Voyage en Orient, Paris, Debécourt, 1836, p. 32-33.

${ }^{9}$ Alphonse de Lamartine, Voyage en Orient, éd. Sarga Moussa, Paris, Champion, 2000, p. 115.

${ }^{10}$ Delaroière, Voyage en Orient, op. cit., p. 34.
} 
Delaroière peut apparaître comme maladroite. À l'inverse, dans la mesure même où il s'installe pour de nombreuses années à la Chambre des députés, Lamartine valorise, quant à lui, la démocratie grecque ${ }^{11},-$ celle qui renaît de ses cendres, à Nauplie, capitale provisoire jusqu'en 1834, mais aussi et surtout celle qui renvoie au modèle athénien ${ }^{12}$ ayant produit de grands hommes politiques, comme Démosthène, incarnant aux yeux de Lamartine le pouvoir légitime de la parole et de la pensée : "L'orateur participe à la fois de la gloire de l'écrivain et de la puissance des masses sur lesquelles et par lesquelles il agit: c'est le philosophe-roi ${ }^{13}$. » La Grèce, cet espace de l'entre-deux, à la fois porte de l'Orient et origine de l'Occident, est ainsi un ailleurs proche, en même temps qu'elle apparaît comme un pont dans l'Histoire, témoin, à travers l'art oratoire, de la continuité entre l'antiquité et le présent.

Après avoir quitté Athènes, les voyageurs s'embarquent pour la Syrie. Suite à une tempête dans les Cyclades, ils font escale à Rhodes : "Voilà donc l'Asie! », s'écrie Lamartine le 23 août $1832^{14}$. Alors que Chateaubriand voyait dans cette île, où résidait une importante communauté grecque, « une petite France au milieu de la Grèce ${ }^{15}$ », ses successeurs sont plutôt sensibles à la diversité ethnique et à la présence des costumes orientaux. Delaroière avoue ainsi que c'est à Rhodes qu'il voit des femmes turques pour la première fois. Mais pour lui, ce « choc culturel » est en réalité la confirmation d'un préjugé anti-islamique déjà bien ancré chez nombre de voyageurs antérieurs, - l'ailleurs, en ce sens, est parfois un « ici » du discours stéréotypé :

Un bandeau blanc descendant jusqu'aux yeux, comme celui des religieuses, cache leur front ; un autre bandeau descend du bas des yeux et cache le reste de la figure : ces yeux isolés au milieu de cette face blanche font un vilain effet ${ }^{16}$.

Et Delaroière de comparer ces femmes voilées à «de grands fantômes blancs ${ }^{17}$ », qui semblent faire écho aux «fantômes ambulans ${ }^{18}$ » que Volney croyait voir, de son côté, lors de son arrivée à Alexandrie en 1783. Comme il le fera souvent dans son récit de voyage, Delaroière stigmatise et creuse la différence en s'appuyant, sans le dire, sur l'autorité de ses prédécesseurs. Lamartine, tout au contraire, s'attache à réduire cette même différence, - au prix, il est vrai, d'une esthétisation de celle-ci. Ainsi décrit-il longuement, à Rhodes, de « ravissantes figures de femmes » largement occidentalisées par les

\footnotetext{
11 «L'attitude des députés est martiale et fière ; ils parlent sans confusion, sans interruption, d'un ton de voix ému mais ferme, mesuré et harmonieux » (Lamartine, Voyage en Orient, op. cit., p. 109).

${ }^{12}$ Voir Claude Mossé, Le « modèle » athénien, Paris, Aubier, 1995.

${ }^{13}$ Lamartine, Voyage en Orient, op. cit., p. 120.

14 Ibid., p. 129.

${ }^{15}$ Chateaubriand, Itinéraire de Paris à Jérusalem [1811], éd. Jean-Claude Berchet, Paris, Gallimard, « Folio », 2005, p. 270.

${ }^{16}$ Delaroière, Voyage en Orient, op. cit., p. 37.

17 Ibid.

${ }^{18}$ Volney, Voyage en Syrie et en Égypte [1787], in Euvres, t. III, Paris, Fayard, 1998, p. 16.
} 
C'est l'œil des femmes d'Italie, mais plus doux, plus timide, plus pénétré de tendresse et d'amour ; - c'est la taille des femmes grecques, mais plus arrondie, plus assouplie, avec des mouvements plus suaves, plus gracieux. - Leur front est large, uni, blanc, poli comme celui des plus belles femmes d'Angleterre ou de Suisse ; mais la ligne régulière, droite et large du nez, donne plus de majesté et de noblesse antique à la physionomie ${ }^{19}$.

Le poète a une vision idéalisante et spiritualisée de ces Orientales, dont on serait bien en peine de dire si elles sont turques, grecques, juives ou autres. Lamartine, comme il le fait presque toujours lorsqu'il se livre à un portrait de femme, a en tête une image intérieure, peut-être inspirée d'un modèle artistique (peinture ou sculpture). Pourtant, on ne peut l'accuser de nier totalement une altérité en la réduisant au même. En effet - et, là encore, c'est une caractéristique de la description lamartinienne -, on est ici dans le presque pareil, à moins qu'il ne s'agisse du légèrement différent. Est-ce une forme d'exotisme, au sens superficiel du terme, qui nous dépayserait à moindre frais ? On a plutôt affaire, chez Lamartine, à un subtil dosage d'ethnographie et d'esthétique, à une observation construite dont on peut dire provisoirement que le but consiste à rapprocher deux mondes censément opposés. En ce sens, les deux Voyage en Orient comparés ont un fonctionnement totalement différent. Malgré un titre semblable renvoyant à un même périple accompli conjointement par deux amis, la représentation que ceux-ci donnent de l'Orient trahit des présupposés antithétiques.

Ces deux regards se rejoignent cependant, de manière ponctuelle, comme on peut le constater à propos de l'épisode du Saint-Sépulcre. Cette église qui contient le tombeau du Christ est, à l'époque, gardée par des Turcs. Lamartine donne de ces derniers une image tout à fait sympathique, contredisant sciemment, comme il le fait systématiquement dans son Voyage en Orient, la turcophobie de Chateaubriand :

Quand je passai, cinq ou six figures vénérables de Turcs, à longues barbes blanches, étaient accroupies sur ce divan recouvert de riches tapis d'Alep; des tasses à café et des pipes étaient autour d'eux sur ce tapis; ils nous saluèrent avec dignité et grâce, et donnèrent ordre à un des surveillants de nous accompagner dans toutes les parties de l'église. Je ne vis rien sur leurs visages, dans leurs propos ou dans leurs gestes, de cette irrévérence dont on les accuse. Ils n'entrent pas dans l'église, ils sont à la porte, ils parlent aux chrétiens avec la gravité et le respect que le lieu et l'objet de la visite comportent. Possesseurs, par la guerre, du monument sacré des chrétiens, ils ne le détruisent pas, ils n'en jettent pas la cendre au vent; ils le conservent, ils y maintiennent un ordre, une police, une révérence silencieuse que les communions chrétiennes, qui se le disputent, sont bien loin d'y garder elles-mêmes 20.

Alors que le narrateur de l'Itinéraire de Paris à Jérusalem, très marqué par le

\footnotetext{
${ }^{19}$ Lamartine, Voyage en Orient, op. cit., p. 131.

${ }^{20}$ Ibid., p. 300-301.
} 
mythe du «despotisme oriental ${ }^{21} »$, voyait les Turcs comme des tyrans et des destructeurs nés, Lamartine inverse totalement cette image pour donner à voir une sorte de tableau orientaliste avec des figures de patriarches tels qu'il en a peut-être vues dans certains tableaux de Rubens ou de Rembrandt. Ni usurpateurs ni violents, ces Turcs sont là, tout simplement, semblant vivre dans une paisible indolence d'où ils ne sortent que pour jouer le rôle d'arbitre face à des communautés chrétiennes dont nombre de voyageurs contemporains disent qu'elles se disputent volontiers l'occupation de l'église du Saint-Sépulcre. Du reste, le très catholique Delaroière le reconnaît lui-même, ce qui, du coup, le conduit à atténuer sa critique des musulmans :

Je fus frappé, dès l'entrée, de voir dans ce temple chrétien des musulmans tranquillement assis sur leur divan, ayant le même calme et la même indolence que partout ailleurs, laissant entrer indistinctement tout le monde, pourvu qu'on leur paie le batchi [le bakchiche, le pourboire]. En réfléchissant à ces Turcs gardiens de cette porte, je finis par me convaincre que la Providence ne pouvait avoir confié en de meilleures mains l'entrée de ce munument. Si les Grecs veillaient à cette entrée, probablement ils la feraient défendre aux Arméniens et aux Latins; si une de ces deux nations pouvait en disposer souverainement, je doute fort qu'elle fût plus tolérante que les Grecs ; chacun voudrait posséder pour soi ce qui est maintenant une propriété commune à tout ce qui porte le nom de chrétien ${ }^{22}$.

Lecteur de Chateaubriand (lequel notait de manière accusatrice, dans l'Itinéraire, à propos de sa seconde visite au Saint-Sépulcre, «je payai de nouveau à Mahomet le droit d'adorer Jésus-Christ $\left.{ }^{23} »\right)$, Delaroière s'en détache momentanément, sans doute après avoir lu le Voyage en Orient de Lamartine, pour concéder, non sans paradoxe ${ }^{24}$, que cette situation d'infériorité dans laquelle se trouvent les chrétiens de Terre sainte est voulue par Dieu même. Mais le reste de son récit de voyage révèle la même distance déjà constatée face à l'orientalisme lamartinien. Nourri de souvenirs littéraires et culturels qui trahissent une vision datée et antagoniste de l'islam, Delaroière présente un contrepoint presque systématique à la turcophilie de son illustre compagnon.

Un dernier exemple illustrera ce positionnement antithétique des deux auteurs : les belles pages où Lamartine se met en scène, regardant le paysage de Constantinople, donnent à voir un poète sensible aux harmonies entre la ville et les collines boisées qui l'entourent, entre le ciel et les flots du Bosphore, entre

\footnotetext{
${ }^{21}$ Voir Jean-Claude Berchet, «Chateaubriand et le despotisme oriental », in Dix-huitième siècle, $\mathrm{n}^{\circ}$ 26, 1994, p. 391-421.

${ }^{22}$ Delaroière, Voyage en Orient, op. cit., p. 109.

${ }^{23}$ Chateaubriand, Itinéraire de Paris à Jérusalem, op. cit., p. 389.

${ }^{24} \mathrm{Il}$ s'attirera d'ailleurs une critique de la part d'un « censeur » catholique, précisément à propos de ce passage, dans un compte rendu par ailleurs globalement favorable : « Le premier passage qui nous a paru peu exact, est celui où M. Delaroière s'applaudit de voir la garde du St. Sépulchre [sic] confiée à des Turcs, qui admettent indistinctement à la célébration des St. Mystères, les catholiques, les hérétiques et les schismatiques. Il craint que si l'une ou l'autre communion en étoit en possession, elle ne repoussât toutes les autres. Cette réflexion inspirée par une espèce de tolérance n'est pas juste. Un chrétien, un catholique qui connaît sa religion, ne sauroit admirer un fait qui déplaît au ciel » (« Voyage en Orient, par M. Delaroière. - Paris 1836 », texte non signé, in Journal historique et littéraire, $1^{\text {er }}$ septembre 1836, p. 235).
} 
les Turcs et une nature qui, pense-t-il, leur est consubstantielle ${ }^{25}$. Delaroière, à l'inverse, renoue une fois encore avec la turcophobie chateaubriandienne (les habitants de la capitale ottomane étaient décrits, dans l'Itinéraire, comme « un troupeau qu'un iman conduit et qu'un janissaire égorge ${ }^{26} » !$ ), mais aussi, plus largement, avec un imaginaire de la cruauté orientale encore véhiculé, à la même époque, dans Les Orientales (1829), - pensons à "Clair de lune », où Hugo évoque « des sacs pesants, d'où partent des sanglots. / On verrait, en sondant la mer qui les promène, / Se mouvoir dans leurs flancs comme une forme humaine ${ }^{27}$ »: ces vers ont peut-être inspiré le «récit» horrifié fait par Delaroière de « deux femmes turques et deux de leurs esclaves, cousues toutes vivantes dans des sacs [et] jetées dans la mer ». On voit que ce type de clichés «orientalistes ${ }^{28}$ » reste présent, alors que l'Empire ottoman est pourtant fortement affaibli, au moins chez certains voyageurs qui s'inscrivent dans la lignée de Chateaubriand et de Volney.

\section{Vers un dialogisme construit}

Il serait toutefois réducteur d'opposer de manière absolue l'orientalisme des deux Voyage en Orient que nous comparons. Ne peut-on pas déceler, chez Delaroière, une forme de dialogisme qui tenterait d'échapper à l'image d'un Orient-objet, image en général dégradée et trop souvent réduite à l'envers d'un moi idéalisé ? S'il n'y a, bien entendu, aucun projet de la sorte que Delaroière chercherait à mettre en œuvre volontairement, on sent que l'expérience du voyage a donné lieu, fût-ce de manière éphémère, à des moments où s'esquisse une forme de réciprocité, en particulier lorsque le voyageur remarque (et note dans son récit) qu'il est lui aussi regardé par ceux-là mêmes qu'il observe, et qu'il se trouve par conséquent, dès lors qu'il est conscient de ce regard, à son tour en position d'étranger, perçu par un sujet mû par une même curiosité. Une telle scène se produit pour la première fois, significativement, peu après le départ de Beyrouth pour Jérusalem, lorsque les voyageurs commencent à se déplacer en caravane, c'est-à-dire selon un régime nomade qui est celui-là même de certaines populations orientales qu'ils côtoient :

[...] tout était tellement nouveau, que nous ne laissâmes échapper aucun détail. Les individus composant les caravanes qui étaient déjà établies près du khan, lors de notre arrivée, nous examinèrent d'abord avec attention, à cause de nos costumes européens et de nos tentes, car les tentes en voyage sont un luxe peu commun dans les caravanes ${ }^{29}$.

De regardé, le voyageur va néanmoins vite reprendre sa posture habituelle de

\footnotetext{
${ }^{25}$ Voir en particulier la page 575 du Voyage en Orient de Lamartine.

${ }^{26}$ Chateaubriand, Itinéraire, op. cit., p. 257.

${ }^{27}$ Victor Hugo, Les Orientales. Les Feuilles d'automne, éd. Franck Laurent, Paris, Librairie Générale Française, «Le Livre de poche classique », 2000, p. 113.

${ }^{28}$ Voir Edward Saï, L'Orientalisme, trad. fr., Paris, Seuil, 1980.

${ }^{29}$ Delaroière, Voyage en Orient, op. cit., p. 52-53.
} 
regardant : «Après le dîner, ne pouvant me détacher de cette scène, je rôdais tout autour des groupes, examinant successivement toutes ces figures étrangères... ${ }^{30}$ » Le verbe examiner, employé pour désigner aussi bien le regard des Orientaux sur les Occidentaux que celui des Occidentaux sur les Orientaux, instaure un début de réciprocité. Sans doute ces deux regards ne sont-ils pas simultanés. Seul le regard du lecteur sur la page, où voisinent ces deux postures d'observation, permet de construire une symétrie visuelle. Mais il n'en reste pas moins que le narrateur prend en compte, l'espace d'un instant, le fait que l'« autre » est bien, lui aussi, un sujet regardant, et qu'il reconnaît implicitement, du même coup, sa propre situation « objectale ».

On ne trouve guère d'exemple, chez Lamartine, de ces scènes de première rencontre où le voyageur se trouve momentanément destabilisé dans sa position de pouvoir. Sans doute est-ce dû au fait que le narrateur lamartinien se présente lui-même comme un "voyant», comme un prophète ${ }^{31}$ dont le regard est d'autant plus averti que son spectre est élargi : "Il faut qu'un homme politique s'éloigne souvent de la scène où se joue le drame de son temps, s'il veut le juger, et en prévoir le dénouement. Prédire est impossible, la prévision n'est qu'à Dieu ; mais prévoir est possible, la prévoyance est à l'homme ", écrit-il lorsqu'il se trouve à Constantinople ${ }^{32}$. Vision téléologique de l'Histoire dont on est aujourd'hui revenu, mais qui montre bien le statut particulier que Lamartine accorde à son propre regard,- un regard forcément clairvoyant, parfois surplombant, et qui ne prend en compte le regard d'autrui que dans la mesure où il s'intègre à une vision de l'avenir prétablie. C'est dire que Lamartine n'exclut pas l'échange des regards, mais que celui-ci est rapidement mis au service d'un sujet voyageur qui a une forte propension à se mettre en scène, - les premiers lecteurs du Voyage en Orient le lui ont d'ailleurs assez reproché. Du reste, il n'est pas difficile de voir que Lamartine, se déplaçant en grand seigneur, avec force chevaux, escorte et domestiques, a sciemment accentué la dimension spectaculaire de cette caravane dont le faste était précisément destiné à impressionner les populations orientales : regarder l'Orient, mais aussi être vu en Orient, et dire qu'on a été vu sur la terre des Prophètes, - telle semble bien avoir été l'une des stratégies privilégiées de construction de l'image de soi véhiculée par le Voyage en Orient.

Examinons, pour terminer, l'un des épisodes les plus étonnants de ce récit, celui de la rencontre avec Esther Stanhope, la nièce du ministre anglais William Pitt. Morte en 1839 à Djoun, un village du Chouf, après avoir vécu plus de trente ans au Liban, elle passait pour avoir des dons divinatoires. Nombre de voyageurs français et européens lui rendirent visite et, comme Lamartine dit dans une formule flatteuse adressée à elle, cette Anglaise quelque peu

\footnotetext{
${ }^{30}$ Ibid., p. 53.

${ }^{31}$ Voir Paul Bénichou, Les Mages romantiques, Paris, Gallimard, 1988, p. 23 et suiv.

${ }^{32}$ Lamartine, Voyage en Orient, op. cit., p. 531.
} 
excentrique constituait l'« une des merveilles de l'Orient » qu'il venait visiter ${ }^{33}$. Cette rencontre, à laquelle Delaroière n'assista pas (il n'en parle donc pas dans son récit), est narrée sur plusieurs pages largement occupées par un dialogue entre deux « voyants », l'un et l'autre attendant d'ailleurs l'arrivée d'un nouveau « $\mathrm{Messie}^{34}$ ». Chacun des protagonistes adopte, de façon symétrique, une double posture, à la fois occidentale et orientale. En effet, lady Stanhope apparaît vêtue d'un "turban blanc» (qui est à vrai dire un élément masculin du costume oriental : cet habillement en cheikh est confirmé par d'autres voyageurs), d'un " schall de cachemire jaune ", d'une « robe turque de soie blanche» recouvrant « une seconde robe d'étoffe de Perse ${ }^{35}$ ». Lamartine, de son côté, est habillé à l'européenne (ce n'est qu'à Damas, ville réputée "fanatique », qu'il revêt provisoirement un costume oriental), mais il rapporte des propos de son interlocutrice le présentant comme un Oriental qui s'ignore : « Vous retournerez dans l'Occident, mais vous ne tarderez pas beaucoup à revenir en Orient : c'est votre patrie. - C'est du moins, lui dis-je, la patrie de mon imagination. - Ne riez pas, reprit-elle ; c'est votre patrie véritable, c'est la patrie de vos pères ${ }^{36} »$. Si lady Stanhope apparaît comme une Occidentale orientalisée, Lamartine se donne à voir comme un homme de l'entre-deux, comme un Occidental qui, voyageant en Orient, ne ferait que revenir à ses origines supposées. Au fond, dans cette représentation très théâtralisée, le voyageur incarne presque à lui tout seul le « rapprochement de l'Orient et de l'Occident ${ }^{37}$ » qu'il appelle de ses vœux. Il y a bien sûr une forme de narcissisme exacerbé dans ce dialogisme très construit, où les « véritables » Orientaux sont évacués du récit au profit de deux personnages qui semblent perpétuellement en représentation. On peut croire, dans un premier temps, avoir affaire à un Orient de comédie, - quoi de plus superficiel et trompeur que le déguisement, l'identité d'emprunt? Pourtant, ces " gesticulations » orientalisantes s'accompagnent, chez Lamartine, d'un point de vue théologique et humaniste tout à fait novateur :

Il me parut que les doctrines religieuses de lady Esther étaient un mélange habile, quoique confus, des différentes religions au milieu desquelles elle s'est condamnée à vivre ; mystérieuse comme les Druzes dont, seule peut-être au monde, elle connaît le secret mystique ; résignée comme le musulman, et fataliste comme lui, avec le juif, attendant le Messie, et avec le chrétien, professant l'adoration du Christ et la pratique de sa charitable morale ${ }^{38}$.

À l'évidence, le narrateur du Voyage en Orient se reconnaît dans ce syncrétisme pré-nervalien, qu'il feint de critiquer par prudence (un mélange confus de doctrines), mais dans lequel il trouve un écho (et une caution) de son propre

\footnotetext{
${ }^{33}$ Ibid., p. 166.

${ }^{34}$ Ibid., p. 171.

${ }^{35}$ Ibid., p. 169.

${ }^{36}$ Ibid., p. 173.

${ }^{37}$ Ibid, p. 457.

${ }^{38}$ Ibid., p. 174.
} 
« rationalisme chrétien ${ }^{39} »,-$ profession de foi personnelle qu'il énonce devant lady Stanhope, mais qu'il ne définit que plus loin dans son récit («Dieu un et parfait pour dogme, la morale éternelle pour symbole, l'adoration et la charité pour culte $\left.{ }^{40} \gg\right)$.

Lamartine cherche toujours, à l'inverse de Chateaubriand ou de Delaroière, le plus grand dénominateur commun entre les religions, donc entre l'ici et l'ailleurs. Dans la mesure même où son voyage en Orient confirme et accentue son éloignement de l'orthodoxie romaine, un respect, voire une compréhension de l'islam (la religion majoritaire dans l'Empire ottoman) deviennent possibles. Relatant sa recontre avec le pacha de Jaffa, Lamartine écrit ainsi : «Je répondis au gouverneur que, bien que je fusse né dans une autre religion que la sienne, je n'en adorais pas moins que lui la souveraine volonté d'Allah; que son culte à lui s'appelait Fatalité et le mien Providence ; mais que ces deux mots différents n'exprimaient qu'une même pensée : Dieu est grand! Dieu est le maître ! Allah kérim $!^{41} »,-$ formule à laquelle fera écho, un peu plus loin, celle du gouverneur de Jérusalem : " tous les hommes sont frères, bien qu'ils adorent, chacun dans leur langue, le Père commun [...]. Allah kérim, Dieu est grand $^{42}$. 》 Or cette expression prend naissance précisément à l'occasion de la rencontre avec lady Stanhope, qui expliquait elle-même à son interlocuteur qu'elle répondait à tout «par le mot sacré des musulmans : Allah kérim $!^{43}$ ».

On voit que ce épisode joue un rôle matriciel dans le Voyage en Orient, du moins sur le plan théologique, car, sur le plan politique, il faut bien avouer qu'il y a chez Lamartine une tentation coloniale qui s'exprime à plusieurs reprises dans le journal de voyage proprement dit, par exemple lorsque le voyageur se trouve à Baalbek («Un aventurier européen, avec cinq ou six mille soldats d'Europe, peut aisément renverser Ibrahim, et conquérir l'Asie... $\left.{ }^{44} \gg\right)$, et surtout dans le « Résumé poltique » qui figure à la fin de l'ouvrage, où le député nouvellement élu envisage sans vergogne un protectorat européen sur un Empire ottoman qu'il imagine déjà « écroulé, divisé et démembré ${ }^{45}$ »; et, malgré l'éloge qu'il continue de prodiguer aux Turcs ( C'est un peuple de patriarches, de contemplateurs, d'adorateurs, de philosophes ${ }^{46} \gg$, etc.), ceux-ci ont pour vocation, selon lui, soit à se fondre dans les populations insurgées, soit à se concentrer à Constantinople et dans quelques régions d'Asie Mineure, où ils seraient soumis à l'influence civilisatrice de l'Europe. Il y a donc incontestablement, dans le « rapprochement de l'Orient et de l'Occident » que

\footnotetext{
${ }^{39}$ Ibid., p. 172.

${ }^{40}$ Ibid., p. 533.

${ }^{41}$ Littéralement : « Dieu est généreux » (ibid., p. 269-270).

${ }^{42}$ Ibid., p. 307.

${ }^{43}$ Ibid., p. 175.

${ }^{44}$ Ibid., p. 425.

${ }^{45}$ Ibid., p. 745.

${ }^{46}$ Ibid., p. 746.
} 
Lamartine considère comme "inévitable ${ }^{47}$ », une double dimension, tout à la fois humaniste et prédactrice, certes généreuse, sur le plan religieux, envers l'islam, mais aussi impitoyable, politiquement, à l'égard de ce que l'on appelle déjà la « Turquie ».

C'est cette ambiguïté que je voudrais souligner pour conclure, en montrant qu'elle est constitutive du Voyage en Orient de Lamartine, à la différence de celui de Delaroière. En effet, chez ce dernier, on ne trouve ni éloge de l'islam (il oppose au contraire la « civilisation chrétienne » à « l'incurie des musulmans ${ }^{48} »$ ), ni tentation impérialiste; rempli de préjugés ( $C$ 'est donc vrai ce qu'on dit des Arabes, qu'il est impossible qu'ils ne mentent pas à l'homme qui les interroge... $\left.{ }^{49} »\right)$, le médecin Delaroière ne développe pas pour autant le moindre projet de démentèlement de l'Empire ottoman. À l'inverse, Lamartine, à la fois poète et député, tient dans son récit de voyage un double discours, l'un, de nature « œcuménique », visant à rapprocher deux grandes religions monothéistes, l'autre, en prise sur l'actualité politique, visant à faire de la France une grande puissance en Méditerranée, - n'oublions pas que les années 1830 marquent le début de la conquête de l'Algérie, que Lamartine soutient, d'ailleurs, comme l'immense majorité de ses contemporains.

On retrouve cette même ambiguité avec le mot patrie, par exemple dans le récit de l'arrivée de Lamartine à Beyrouth, qualifiée précisément de «nouvelle patrie $^{50} »$, qui fascine par sa différence orientale donnant à rêver (tous les clichés romantiques que Segalen condamnera dans son Essai sur l'exotisme y passent : palmiers, harems, chameaux...), mais dont le drapeau flottant sur le consulat français, lui-même situé en position « impériale », au sommet de la ville, rassure le voyageur en terre étrangère. Quant au dialogue avec lady Stanhope, il révèle un véritable dédoublement sémantique de ce même mot de patrie. Le poète romantique se met en scène, tel un nouveau Byron, comme celui qui non seulement voyage et franchit des limites, mais qui fait aussi de l'Orient, cet ailleurs désiré, une nouvelle patrie littéraire. Toutefois, son interlocutrice (dont le narrateur fait une prophétesse) entend les choses autrement: l'Orient, rappelle-t-elle, est origine (c'est en effet le sens étymologique du mot), et, à ce titre, y revenir n'est pas vivre une expérience d'altérité, mais au contraire retrouver, sur cette terre qui a nourri la Bible, le Livre qui constitue le socle même de l'Occident judéo-chrétien. Quelle est, alors, la " grande mission » que la France est censée accomplir, et à laquelle Lamartine devra activement contribuer, comme lady Stanhope croit pouvoir l'assurer à son visiteur ? La formule n'est pas transparente, comme il se doit venant d'une Sibylle, mais on voit bien qu'elle peut se prêter à une interprétation à laquelle le discours colonial

\footnotetext{
${ }^{47}$ Ibid., p. 457.

${ }^{48}$ Delaroière, Voyage en Orient, op. cit., p. 136 et 133.

${ }^{49}$ Ibid., p. 148.

${ }^{50}$ Lamartine, Voyage en Orient, op. cit., p. 139.
} 
à venir ne manquera pas de recourir ${ }^{51}$.

Quoi qu'il en soit, ce qui se joue dans le voyage en Orient, au XIX ${ }^{\mathrm{e}}$ siècle, est bien une possible reconfiguration de l'opposition traditionnelle entre l' « ici » et l'« ailleurs ». Si celle-ci est largement maintenue chez Delaroière, elle est au contraire destabilisée chez Lamartine, pour qui l'expérience du monde oriental donne lieu, dans son récit, à différentes attitudes, qui vont de la séduction exotique au rejet de l'ailleurs comme envers négatif de l'ici, en passant par des moments de communion spirituelle (l'islam comme religion sœur du christianisme), ou encore des rencontres plus complexes qui font de l'« autre » un hybride renvoyant en partie seulement au même, - rappelons-nous ces Orientales, à Rhodes, comparées successivement à des Italiennes, à des Grecques, à des Anglaises et à des Suissesses, mais dont les traits du visage ont néanmoins une spécificité qui les distingue de ces différents «modèles». Ce caractère instable de l'ailleurs rend du même coup l'ici inassignable géographiquement.

Nous sommes aujourd'hui, à l'évidence, redevables à la littérature romantique (et au rôle que certains récits de voyage en Orient y ont joué) de ces déplacements.

\footnotetext{
${ }^{51}$ Michel Butor le dénoncera clairement, au début d'Égypte, texte paru peu après l'affaire de Suez : «car, je puis le dire (j'use de ce mot si détourné de sa racine, si associé ici désormais à tant d'horribles et insinuantes duperies, avec les plus grandes précautions et parce qu'il n'y en a pas d'autre), l'Égypte a été pour moi comme une seconde patrie... » (Le Génie du lieu [1958], Paris, Grasset, « Les Cahiers Rouges », 1994, p. 110).
} 